\title{
Etude expérimentale du transport de sable fin en suspension sous l'action de la houle et du courant
}

\author{
C. Villaret et B. Latteux
}

EDF, Laboratoire National d'Hydraulique

\section{Introduction}

La prédiction du transport solide en milieu littoral est toujours un thème d'actualité au Laboratoire National d'Hydraulique, en raison des nombreuses applications pratiques à des problèmes liés à l'aménagement du littoral et à la protection de l'environnement. La houle et les courants jouent des rôles complémentaires au niveau de la mise en suspension et du transport solide. Un programme expérimental a été mis sur pied dans le cadre des projets européens "MAST G6/G8 Morphodynamics ", en vue d'étudier le transport en suspension de sable fin non cohésif sous l'action du courant, de la houle régulière, ainsi que sous l'action combinée de la houle et du courant. Pour les paramètres hydrosédimentaires considérés, des formes de fond de dimensions relativement petites par rapport aux dimensions caractéristiques de l'écoulement sont générées spontanément à la surface du lit sédimentaire. L'objectif principal des expériences présentées ci-après est d'étudier l'influence de ces rides sur l'écoulement au fond et sur le transport solide en suspension pour les différents régimes hydrodynamiques considérés.

L'influence de rides sur l'écoulement moyen est généralement pris en compte par une augmentation du coefficient du rugosité équivalente qui intervient dans la loi classique du profil logarithmique des vitesses. Ce coefficient peut être prescrit en fonction des dimensions des rides à l'équilibre selon une des nombreuses formules semi-empiriques proposées dans la littérature (e.g. RAUDKIVI (1990)). Ces formules sont cependant en général obtenues pour des régimes uniformes et stationnaires, dans le cas idéal de rides bidimensionnelles pleinement développées. L'influence d'un courant non permanent sur les formes de fond est un domaine encore peu exploré. En régime oscillatoire, de nombreuses expérimentations intéressantes ont été recensées, depuis les premières réalisées en houle régulière seule (VINCENT, 1957), ainsi qu'en houle et courant superposés ( $c f$. VAN RuN, 1990). Les processus fortement non linéaires qui régissent le transport en suspension sous l'action combinée de la houle et d'un courant, soit superposé à la houle, soit généré par la houle, sont cependant encore mal connus.

Les essais engagés au LNH tentent de compléter les essais rapportés dans la littérature par un choix approprié des paramètres hydrosédimentaires. Une première série d'essais a été réalisée en courant seul. On étudie le développement de rides à partir d'un fond plat, puis l'effet de la renverse ainsi que l'effet d'une accélération ou d'une diminution brusque du courant sur les dimensions des rides et sur leur temps d'adaptation. Une seconde série d'expériences a été réalisée en houle régulière seule ainsi qu'en houle et courant combinés, dans le but de mettre en évidence l'influence du mouvement oscillatoire sur les caractéristiques des rides et sur le transport en suspension.

\section{Dispositif expérimental}

Les expériences ont été réalisées dans un canal à houle de $80 \mathrm{~m}$ de longueur, $1 \mathrm{~m} 50$ de largeur et $1 \mathrm{~m} 50$ de

\section{Experimental study of fine suspended sediment transport by waves and currents}

\begin{abstract}
Open channel experiments have been performed in order to study the development of ripples and the transport in suspension of fine sand, by currents, regular waves, and superposed waves and currents. Measurements of instantaneous velocities and concentrations were carried out in order to calculate fluxes of suspended material. Ripples mean dimensions could be obtained from a statistical analysis of bed profiler data during both equilibrium and transition regime.
\end{abstract}


hauteur, équipé d'un côté d'un batteur de type piston et de l'autre, d'une plage d'amortissement. Aux deux extrémités sont situées des bouches à courant ( $c f$. fig. l). Un courant réversible est généré par une pompe de $1000 \mathrm{l} / \mathrm{s}$. Une partie du débit total, réglable au moyen d'un partiteur, alimente le canal. Le canal est surélevé par une plate-forme centrale de $40 \mathrm{~cm}$ de hauteur sur $20 \mathrm{~m}$ de longueur, avec des pentes latérales de $2 / 15$, ce qui permet d'assurer le piégeage des sédiments de part et d'autre de la plate-forme. Un lit de sédiments fins non cohésifs de diamètre moyen $d_{50}=90 \mu \mathrm{m}$ est disposé sur $10 \mathrm{~cm}$ d'épaisseur au-dessus de la plate-forme. Les appareils de mesure sont installés sur un chariot mobile. La section de mesure est située au centre de la plate-forme. Pour plus de détails, le lecteur peut se référer aux rapports LNH (Villaret et Perrier (1992), Villaret (1994)).

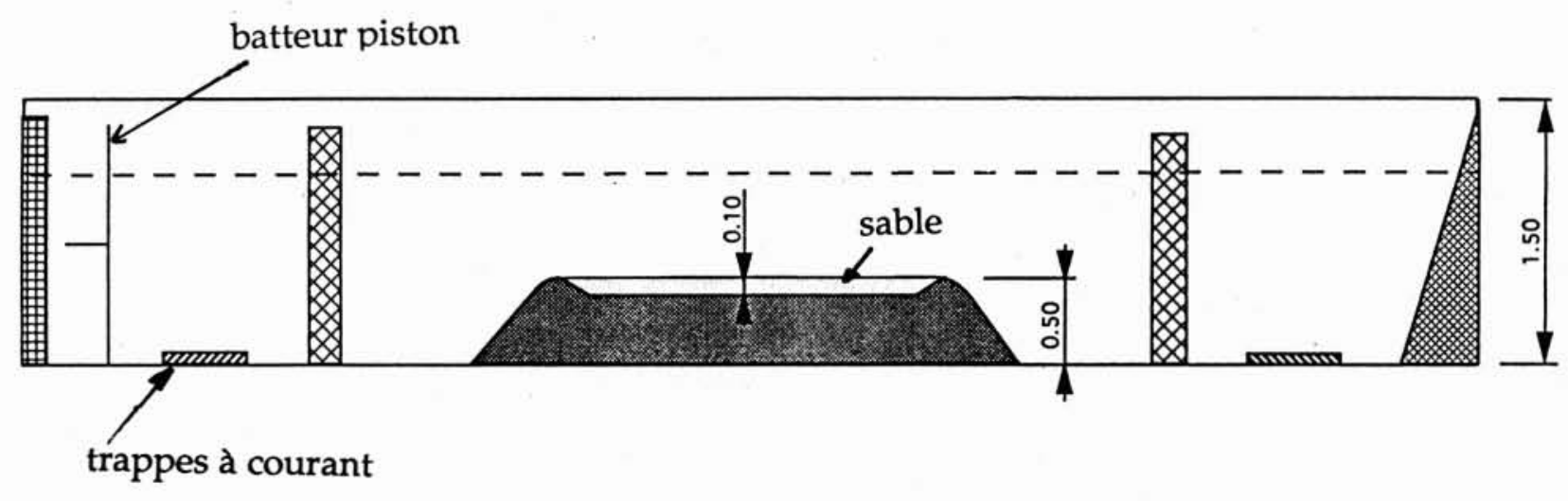

1. Schéma du canal à houle et courant en vue de côté.

\section{Mesures}

Les vitesses instantanées sont mesurées à l'aide de sondes ultrasons unidimensionnelles situées à $45^{\circ}$ par rapport à l'écoulement (MINILAB). Une sonde optique (OPCON) est utilisée pour les mesures de concentrations instantanées. Les mesures sont réalisées en 8 points sur la verticale de $z=0,2 \mathrm{~cm}$ à $z=10 \mathrm{~cm}$. La position des instruments est repérée visuellement par rapport au niveau des crêtes des rides. Les mesures sont échantillonnées à $100 \mathrm{~Hz}$, la durée de chaque enregistrement étant de $75 \mathrm{~s}$ environ, soit une cinquantaine de vagues.

Le transport total (charriage et suspension) est estimé à partir des volumes de sable récolté dans les pièges à sédiments. Le transport en suspension $Q_{s}$ est mesuré directement par intégration sur la verticale des mesures instantanées des flux de concentration :

$$
Q_{s}=\int_{0}^{h}\langle u(t) c(t)\rangle \mathrm{d} z .
$$

En courant seul, le transport par les fluctuations turbulentes étant en général considéré comme négligeable, $Q_{s}$ est égal au transport des concentrations moyennes $C=\langle c(t)\rangle$ par le courant moyen $U=\langle u(t)\rangle$ :

$$
Q_{c}=\int_{0}^{h}\langle u\rangle\langle c\rangle \mathrm{d} z .
$$

En houle seule ainsi qu'en houle et courants superposés, le transport en suspension $Q_{s}$ est modifié par une contribution due au mouvement périodique $Q_{w}\left(=Q_{s}-Q_{c}\right)$ qui représente les corrélations entre les composantes périodi- ques des vitesses $u_{w}$ et des concentrations $c_{w}$ :

$$
Q_{w}=\int_{0}^{h}\left\langle u_{w}(t) c_{w}(t)\right\rangle \mathrm{d} z .
$$

Les composantes périodiques $u_{w}$ et $c_{w}$ sont obtenues par une opération de moyenne par phase sur les mesures instantanées.

En courant seul, des mesures du niveau moyen de la surface libre sont réalisées à l'aide de 4 capteurs répartis le long de la plate-forme de mesure. La pente moyenne de la surface libre $I$ est obtenue par régression linéaire sur les mesures des capteurs. La vitesse de frottement totale $\left(u_{*}=\sqrt{\tau_{b} / \rho}\right)$, où $\tau_{b}$ est le frottement exercé par le courant moyen sur le fond, peut être calculée en fonction de $I$ en écrivant l'équilibre entre les forces de gravité et de frottement sur le fond :

$$
u_{.}=\sqrt{g h I} .
$$

Le profil logarithmique des vitesses, supposé valable sur toute la hauteur d'eau $h$, après intégration sur la verticale, s'écrit :

$$
\frac{U_{h}}{u_{*}}=\frac{1}{K} \operatorname{Ln}\left(\frac{h}{k_{s}}\right)+6
$$

où $K$ est la constante de Karman et $U_{h}$, la vitesse moyenne intégrée sur la verticale. La relation (5) est utilisée pour estimer le coefficient de rugosité équivalente $k_{s}$ en fonction de $U_{h}$ et de $u^{*}$ calculée par la relation (4). En accord avec l'hypothèse de profil logarithmique des vitesses, la vitesse moyenne sur la verticale $U_{h}$ est 
mesurée directement à la distance $z_{t}=h / e$ du fond (soit $z_{r}=11 \mathrm{~cm}$ pour les expériences en courant).

On utilise un palpeur électromagnétique pour les relevés de fond. Une analyse statistique des différents relevés sur une section longitudinale de 2 à $3 \mathrm{~m}$ permet de déterminer les dimensions moyennes des rides et leur évolution dans le temps. Les prises de vue vidéo et photographiques au cours de l'expérience donnent des indications sur les évolutions rapides des formes de fond, en particulier pendant les régimes transitoires.

\section{Conditions expérimentales}

Les essais en courant seul ont été réalisés avec une faible hauteur d'eau $(h=30 \mathrm{~cm})$ au-dessus de la plate-forme. afin de minimiser les courants secondaires. A l'instant initial $(t=0)$, le courant est établi et maintenu constant par paliers de 2 heures environ, temps au bout duquel les rides ont atteint leur dimension d'équilibre. L'intensité du courant est réglée de 20 à $50 \mathrm{~cm} / \mathrm{s}$. Une première série d'expériences (série (a)) est réalisée pour observer le développement de rides à partir d'un fond plat. Dans une seconde série d'expériences (série (b)), on part d'un fond préalablement ridé par un courant de même intensité mais de direction opposée, pour simuler l'effet de la renverse : on étudie aussi de manière similaire l'effet d'une accélération (série (c)) ou décélération brusque du courant (série (d)). Les paramètres hydrodynamiques des différents essais sont récapitulés dans le tableau la (séries (a), (b), (c) et (d)).

Tableau 1. - Paramètres hydrodynamiques et dimensions des rides à l'équilibre. 1.a : Essai en courant seul. (Série (a): Départ fond plat ; (b) Renverse ; (c) Accélération ; (d) Décélération). 1.b : Essais en Houle $(\mathrm{H})$; Houle + Courant $(\mathrm{H}+\mathrm{C})$; Houle - Courant $(\mathrm{H}-\mathrm{C})(\mathrm{h}=60 \mathrm{~cm})$.

(a)

\begin{tabular}{|c|c|c|c|c|c|c|c|c|}
\hline $\begin{array}{c}\text { Test } \\
\mathbf{N}^{\circ}\end{array}$ & $\begin{array}{c}\mathbf{h} \\
(\mathbf{c m})\end{array}$ & $\begin{array}{c}\mathbf{U}_{\mathbf{h}} \\
(\mathbf{c m} / \mathbf{s})\end{array}$ & $\begin{array}{c}\mathrm{I} \\
\mathbf{x} 10^{-4}\end{array}$ & $\begin{array}{c}\mathbf{u}_{*} \\
(\mathbf{c m} / \mathbf{s})\end{array}$ & $\begin{array}{c}\mathbf{k}_{\mathbf{s}} \\
(\mathbf{c m})\end{array}$ & $\begin{array}{c}\lambda \\
(\mathbf{c m})\end{array}$ & $\begin{array}{c}\delta \\
(\mathbf{c m})\end{array}$ & $\delta / \lambda$ \\
\hline 107 & 29.6 & 26.6 & 1.1 & 1.9 & 0.85 & 6.9 & .45 & .07 \\
\hline 116 & 29.7 & 28.4 & 1.6 & 2.2 & 1.8 & 7.3 & 0.65 & .085 \\
\hline 110 & 29.7 & 28.5 & 1.4 & 2.0 & 1.2 & 6.4 & 0.6 & .09 \\
\hline 138 & 29.6 & 29.2 & 1.9 & 2.3 & 2.2 & 8.7 & .85 & .10 \\
\hline 102 & 28.0 & 35.9 & 2.65 & 2.75 & 1.7 & 7.9 & 0.75 & .09 \\
\hline 134 & 29.2 & 46.8 & 5.6 & 4.0 & 3.0 & 10.0 & .8 & .08 \\
\hline 139 & 29.5 & 25.7 & 1.2 & 1.8 & 1.3 & 7.5 & 0.8 & .11 \\
\hline 104 & 29.8 & 28.3 & 1.6 & 2.2 & 1.7 & 8.4 & 0.8 & .09 \\
\hline 135 & 29.4 & 48.6 & 5.6 & 4.0 & 2.8 & 10.9 & 1.1 & .10 \\
\hline 108 & 29.4 & 37.1 & 2.9 & 2.9 & 2.0 & 8.6 & .7 & .08 \\
\hline 117 & 29.5 & 36.7 & 3.4 & 3.1 & 2.9 & 9.6 & .9 & .10 \\
\hline 111 & 29.5 & 37.9 & 3.8 & 3.3 & 3.3 & 10.0 & 1.1 & .11 \\
\hline 109 & 29.4 & 27.3 & 1.7 & 2.2 & 2.3 & 9.4 & 0.9 & .10 \\
\hline 112 & 29.5 & 29.3 & 1.9 & 2.3 & 2.3 & 10. & 1.0 & .10 \\
\hline
\end{tabular}

(H)

\begin{tabular}{|c|c|c|c|c|c|c|c|c|}
\hline $\begin{array}{c}\text { Test } \\
\mathbf{N}^{\circ}\end{array}$ & $\begin{array}{c}\mathrm{T} \\
(\mathbf{s})\end{array}$ & $\begin{array}{c}\mathrm{Hi} \\
(\mathbf{c m})\end{array}$ & $\begin{array}{c}\mathrm{Uo} \\
(\mathbf{c m} / \mathbf{s})\end{array}$ & $\begin{array}{c}\mathbf{A o} \\
(\mathbf{c m})\end{array}$ & $\begin{array}{c}\mathbf{U} \\
(\mathbf{c m} / \mathbf{s})\end{array}$ & $\begin{array}{c}\lambda \\
(\mathbf{c m})\end{array}$ & $\begin{array}{c}\delta \\
(\mathbf{c m})\end{array}$ & $\delta / \lambda$ \\
\hline 35 & 1.5 & 23.2 & 30 & 7 & -5 & 5.0 & 0.7 & .14 \\
\hline 39 & 2 & 23.2 & 37 & 12 & -6 & 6.1 & 0.75 & .12 \\
\hline 42 & 2.5 & 25.0 & 44 & 17.5 & -6 & 9.4 & 0.75 & .08 \\
\hline 36 & 1.5 & 18.8 & 24. & 6 & 17 & 5.5 & 0.65 & .12 \\
\hline 40 & 2 & 21.0 & 34 & 11 & 17 & 5.7 & 0.7 & .12 \\
\hline 77 & 2.5 & 17.8 & 31. & 12.5 & 17 & 6.8 & 0.85 & .125 \\
\hline 38 & 1.5 & 17.5 & 23 & 5 & -17 & 5.2 & 0.6 & .12 \\
\hline 41 & 2 & 21.1 & 34. & 11 & -19 & 6.4 & 0.75 & .12 \\
\hline 45 & 2.5 & 21.0 & 37 & 15 & -18 & 6.6 & 0.7 & .11 \\
\hline
\end{tabular}


Les expériences en houle seule $(\mathrm{H})$, ainsi qu'en houle et courants superposés $(H+C)$ et en houle contrecourant $(H-C)$, sont réalisées avec une hauteur d'eau $h=60 \mathrm{~cm}$ au-dessus de la plate-forme. La période $T$ de la houle varie de 1,5 à $2,5 \mathrm{~s}$, pour une hauteur de houle incidente $H_{i} \approx 25 \mathrm{~cm}$. Le courant moyen superposé à la houle est de l'ordre de $18 \mathrm{~cm} / \mathrm{s}$. Le signal de houle est enregistré au moyen de trois capteurs situés au centre de la plate-forme afin de séparer les composantes incidente et réfléchie. Le coefficient de réflexion est inférieur à $10 \%$. Les vitesses orbitales $U_{0}$ et l'amplitude $A_{0}$ du mouvement orbital sont calculées suivant la théorie de la houle linéaire. Les paramètres hydrodynamiques des différents essais en houle $(\mathrm{H})$ ainsi qu'en houle et courant combinés $(H+C, H-C)$ sont indiqués dans le tableau l.b.

\section{Dimensions des rides à l'équilibre}

Courant seul: le temps de développement des rides à partir d'un fond plat (série (a)) est de l'ordre de 2 heures et diminue lorsque l'intensité du courant augmente. Le temps de développement des rides est aussi influencé par la régularité du régime transitoire ainsi que par les conditions de préparation du lit sédimentaire. Dans le cas de la renverse (série (b)), le temps d'adaptation des rides est très court, de l'ordre d'une dizaine de minutes.

A l'équilibre, les rides sont asymétriques, la pente la plus forte étant située à l'aval de la ride par rapport au courant. En vue de dessus, les rides sont quasi-bidimensionnelles pour les courants de faible intensité et s'organisent en un schéma tridimensionnel lorsqu'on augmente l'intensité du courant ( $c f$. fig. 2.a). Après diminution du courant, les rides conservent en général le schéma tridimensionnel obtenu lors du palier précédent. La longueur moyenne des rides à l'équilibre est de l'ordre de $\lambda \approx 10 \mathrm{~cm}$, pour une hauteur moyenne $\delta=1 \mathrm{~cm}$. Les mesures des dimensions des rides sont en bon accord avec les observations rapportées dans la littérature: $\lambda \approx 1000 d_{511}$. pour une cambrure moyenne $\delta / \lambda \approx 0,1$.

Les dimensions des rides à l'équilibre ont tendance à augmenter avec le nombre de Reynolds des particules $R^{*}=\frac{d_{50} u^{*}}{v}$ (cf. fig. $3 a$ ). L'influence de l'état initial, à partir d'un fond plat ou ridé, ne semble pas avoir d'influence sensible sur les dimensions des rides à l'équilibre. En particulier, lors de la renverse, le changement d'asymétrie des rides s'effectue à longueur d'onde et hauteur constantes. Il est par contre intéressant de noter que les dimensions obtenues après décélération brusque du courant (série (d)) sont légèrement supérieures à celles obtenues pour les séries (a) et (b) correspondant à un même nombre de Reynolds. Ce résultat indique que les rides générées par un courant d'intensité plus forte sont maintenues après diminution du courant.

Pour les expériences en houle seule ainsi qu'en houle et courant superposés, les rides atteignent rapidement leurs dimensions d'équilibre en moins de $15 \mathrm{~min}$. A l'équilibre, les rides sont symétriques avec une crête pointue en houle seule ( $c f$. fig. 2b). Les formes de fond obtenues en houle et courant superposés sont semblables à celles obtenues

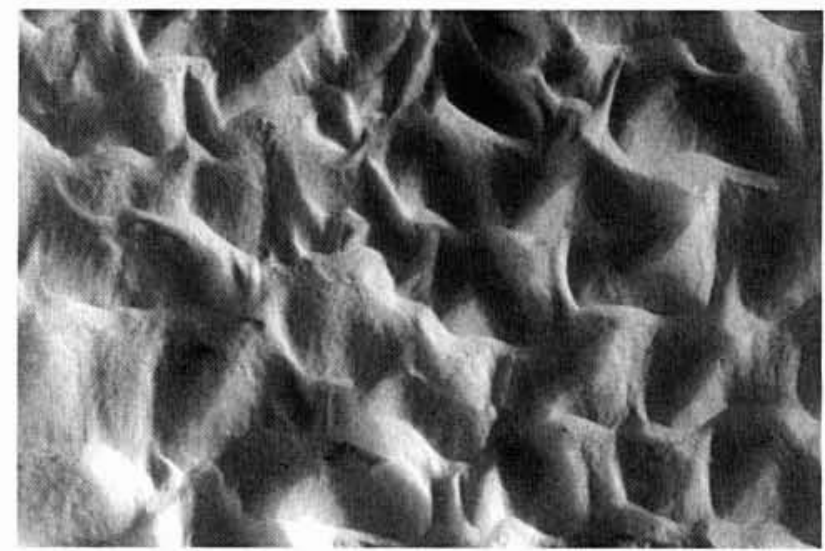

a)

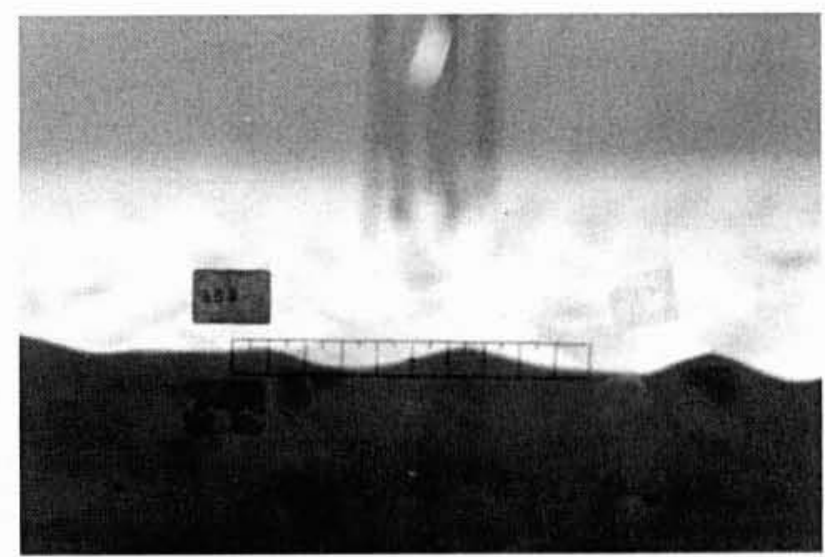

b)

2. Rides à l'équilibre:

a) Vue de dessus en courant seul (Test 134).

b) Vue de côté sous la houle (Test 35).

en houle seule, ce qui indique que, dans la plage de régimes étudiés, l'influence de la houle est prépondérante. La longueur moyenne des rides $\lambda$ est comprise entre 5 et $9,5 \mathrm{~cm}$, pour une hauteur $\delta$ de l'ordre de 0,7 à $0,8 \mathrm{~cm}$. La longueur des rides augmente avec la période de la houle. Sur la figure $3 b$, le rapport $\lambda / A_{0}$ est tracé en fonction du nombre de mobilité $M$, défini par:

$$
M=\frac{U_{0}^{2}}{(s-1) g d_{50}}
$$

avec $s=2,65$ pour la densité des grains. Le modèle de Nielsen (1981) qui s'écrit:

$$
\begin{array}{lll}
\frac{\lambda}{A_{0}}=2,2-0,345 M^{0,34} & \text { pour } & M>20 \\
\frac{\lambda}{A_{0}}=1,3 & \text { pour } & M<20
\end{array}
$$

est en bon accord avec les mesures expérimentales ( $c f$. fig. $3 b$ ). 
$\lambda(\mathrm{cm})$

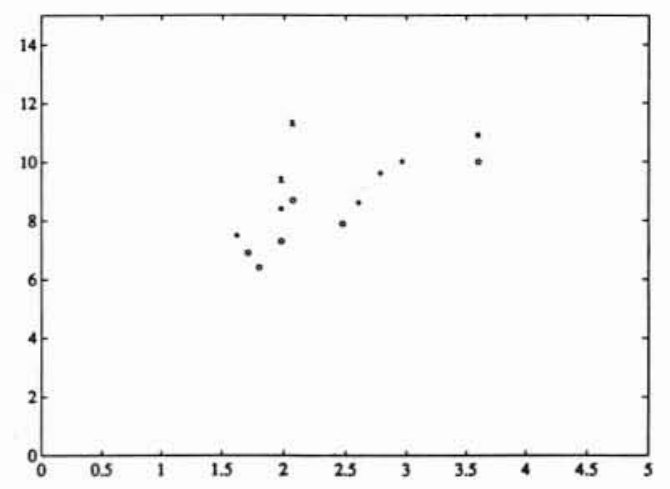

R.

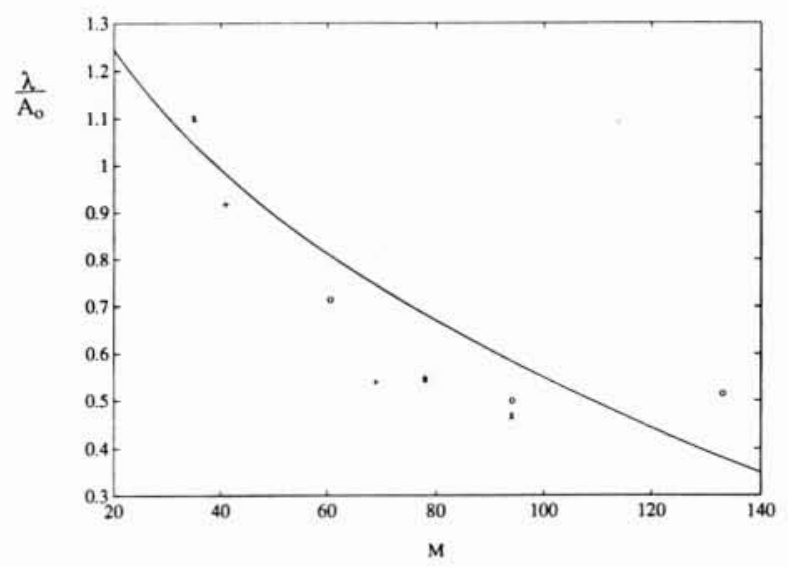

3. Longueur d'onde des rides à l'équilibre:

a) Influence du nombre de Reynolds $R_{*}=\frac{d_{50} u_{*}}{\nu}$.

Courant seul (Series $(a): \alpha o \cdots:(b): * * *:(c): \alpha+\cdots$; (d) : $\alpha x \cdots$.

b) Influence du nombre de mobilitế $M=\frac{U_{0}^{2}}{(s-1) g d_{50}}$

$(H: \cdots 0 \cdots: H+C: *+\cdots, H-C: \approx x \cdots$, equation (7): "-

\section{Rugosité équivalente (Courant seul)}

Les résultats obtenus pour les coefficients de rugosité sont indiqués dans le tableau la. Le rapport moyen $k_{s} / \delta$ varie entre 1,5 et 4 , avec une valeur moyenne de 2,6 . Les résultats sont comparés sur la figure 4 avec la formule semi-empirique de RAUDKIVI (1990) :

$$
\frac{k_{s}}{\delta}=20 \frac{\delta}{\lambda} \text {. }
$$

Cette formule a tendance en moyenne à sous-estimer les valeurs expérimentales, tout en respectant le bon ordre de grandeur. Ce résultat peut être lié au caractère fortement tridimensionnel des formes de fond en particulier pour les courants d'intensité forte.

\section{Transport en suspension :}

Pour les expériences en courant seul, le transport en suspension est très faible et n'a pu être mesuré précisé-

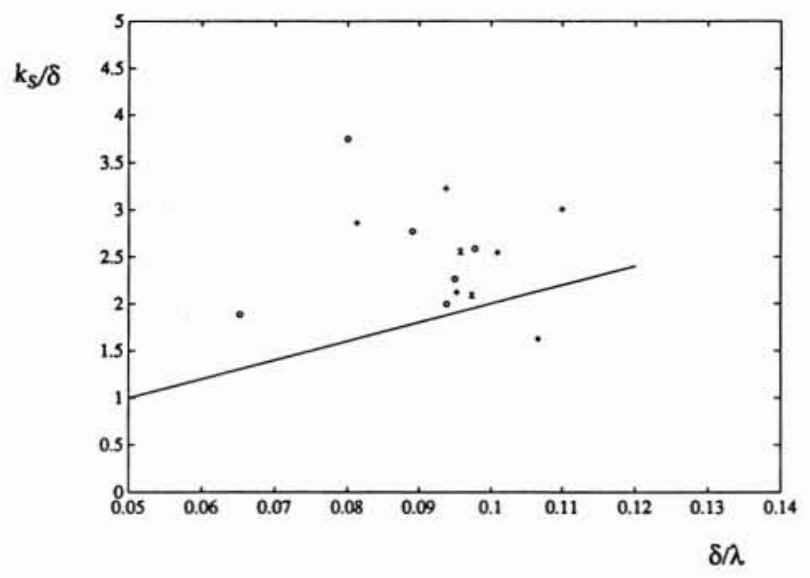

4. Coefficient de rugosité en fonction des dimensions des rides à l'équilibre. Courant seul (Series $(a): \propto 0 n ;(b): \approx *_{\infty} ;(c)$ : $\approx+\cdots ;(d): \alpha x \cdots$, equation $(\delta): \alpha-\cdots$ :

ment. Pour les expériences en houle seule ainsi que pour les essais en houle et courant superposés, le transport est intense et se fait essentiellement en suspension. La souscouche concentrée d'épaisseur proportionnelle à la hauteur des rides $(\approx 1-2 \delta)$, est une zone extrêmement dynamique. On peut observer à chaque demi-période la génération de vortex par les phénomènes de décollement à l'aval des rides et leur éjection au-dessus des crêtes au moment de la renverse. Ces tourbillons, dont le diamètre est de l'ordre de quelques hauteurs de rides, sont les agents essentiels de mise en suspension puis de transport des sédiments. Les résultats obtenus pour les expériences en courant oscillatoire sont indiqués dans le tableau 2 et sont interprétés en terme de dynamique des tourbillons.

Tableau 2. - Taux de transport en suspension: $(Q s=Q w+Q c)$; (Les unités sont en $\mathrm{m}^{2} / \mathrm{s}$.)

(H)

\begin{tabular}{|c|c|c|c|}
\hline $\begin{array}{c}\text { Test } \\
\mathbf{N}^{\circ}\end{array}$ & $\begin{array}{c}\mathbf{Q}_{\mathrm{c}} \\
\times 10^{-6}\end{array}$ & $\begin{array}{c}\mathbf{Q}_{\mathbf{w}} \\
\times 10^{-6}\end{array}$ & $\begin{array}{c}\mathbf{Q}_{\mathbf{s}} \\
\times 10^{-6}\end{array}$ \\
\hline 35 & 0.8 & 3.8 & 4.5 \\
\hline 39 & 0.1 & 4.2 & 4.3 \\
\hline 42 & .4 & 6. & 6.4 \\
\hline 36 & 5.3 & -0.8 & 4.5 \\
\hline 40 & 5.1 & -2.1 & 3.0 \\
\hline 77 & 6.3 & -1.4 & 4.7 \\
\hline 38 & -1.1 & 1.4 & 0.3 \\
\hline 41 & -2.4 & 5.5 & 3.1 \\
\hline 45 & -2.3 & 3.6 & 1.3 \\
\hline
\end{tabular}

Dans le cas de la houle seule $(H)$, on trouve que le transport dû à la houle est dans le sens de propagation de la houle $\left(Q_{s}=Q_{w}>0\right)$. Ce résultat est en contradiction avec les observations précédentes de SATO et HORIKAWA (1986) qui ont trouvé un transport $Q_{s}<0$ pour une houle de Stokes asymétrique, générée en tunnel hydrodynami- 


\section{VILLARET, B. LATTEUX}

que. En canal à houle, les mesures de vitesse mettent en évidence la présence d'un courant moyen dirigé dans le sens de l'écoulement au niveau du fond et opposé en surface, en accord qualitatif avec le modèle théorique de LONGUET-HIGGINS (1953). La composante $Q_{c}$ du transport par le courant moyen reste relativement faible $\left(Q_{c} \approx 0\right)$. Cependant, les résultats obtenus indiquent que le courant moyen généré par la houle, en interagissant avec les tourbillons du mouvement périodique, joue un rôle déterminant sur la direction du transport par la composante périodique $Q_{u}$.

En houle et courant superposés $(H+C ; H-C)$, on trouve que la composante due au mouvement oscillatoire $Q_{w}$ s'oppose systématiquement au mouvement moyen $Q_{c}$; la composante périodique du transport a donc pour effet de diminuer l'advection par le courant moyen. Ce résultat, a priori surprenant, est en accord qualitatif avec les observations précédentes de DAVIES et MURRAY (1990). La présence du courant moyen a pour effet de dissymétriser les tourbillons générés par l'écoulement périodique, avec renforcement des tourbillons transportés dans le sens opposé au courant moyen.

\section{Conclusions}

Dans le cas d'écoulements permanents sur fond ridé asymétrique, le frottement moyen exercé par le courant sur le fond peut être modélisé par l'intermédiaire d'un coefficient de rugosité équivalente proportionnel à la hauteur des rides. Le temps de formation des rides à partir d'un fond plat est de l'ordre de $2 \mathrm{~h}$ et diminue lorsque l'intensité du courant augmente. Lors de la renverse, le temps d'adaptation des rides est très court. Le transport total, très faible, n'a pas pu être évalué avec précision.

Le transport de sédiments en houle seule est dans le sens de propagation de la houle, tandis qu'en houle et courant superposés, la composante du transport liée au mouvement oscillatoire s'oppose systématiquement au transport par le courant moyen. Ces résultats, qui illustrent bien la complexité des interactions non linéaires entre les mouvements moyen et périodique, sont interprétés en terme de dynamique des tourbillons. Certains mécanismes, notamment en ce qui concerne le rôle du courant de masse induit par la houle, méritent d'être éclaircis par une étude hydrodynamique détaillée.

\section{Références}

LONGUET-HigGings M.S. (1953). - Mass transport in water waves, Phil Trans Roy Soc Lond, Vol. 245, pp. 535581.

Murray P.B., Davies A.G. (1990). - Sediment pick-up in wave and current flows. Sand Transport in Rivers, Estuaries and the Sea., Proceedings of the EUROMECH 262 Colloquium, 37-43.

NIELSEN P. (1981). - Dynamics and geometry of wave generated ripples, J. Geophysical Research, Vol. 86, N" C7, pp. 6467-6472.

Raudkivi A.J. (1990). - Loose Boundary Hydraulics, Pergamon Press (3rd edition).

Sato S., Horikawa K. (1986), - Laboratory studies on sand transport over ripples due to asymmetric oscillatory flows, 20th Coastal Engineering Conference, Taipeh, Taiwan.

VAN RuN L.C. (1990). - Sediment transport in combined currents and waves, Sand Transport in Rivers, Estuaries and the Sea, Proceedings of the EUROMECH 262 Colloquium, 3-15.

Villaret C., Latteux B. (1992). - Transport of Fine Sand by Combined Waves and Current : an Experimental Study, 23rd Int. Conf. on Coastal Engineering, Venise.

Villaret C., Perrier G. (1992). - Transport of Fine Sand by Combined Waves and Current : an Experimental Study, Rapport EDF-LNH HE-42/92.68.

VILLARET C. (1994). - Experimental investigation of ripple formation and development and their influence on equivalent roughness coefficients, Rapport LNH-EDF HE$42 / 94$.

VINCENT G.E. (1957). - Contribution to the study of sediment transport on a horizontal bed due to wave action. Proc. 6th Coastal Eng. Conf., Florida.

\section{Remerciements}

L'étude a été réalisée dans le cadre du projet européen MAST G6/G8 "Coastal Morphodynamics", financée en partie par la CEE et par le STCPMVN du Secrétariat à la Mer. Les mesures ont été effectuées par Messieurs J.M. Menon et D. Marchal. 